\title{
Epidemiología de las intoxicaciones agudas por sustancias de abuso en Urgencias. Estudio descriptivo en el área IV de Asturias
}

\section{Epidemiology of acute poisoning by substances of abuse in the Emergency Department. Descriptive study in District IV of Asturias}

\author{
Ana Fernández-Ibáñez*, Rodrigo Ugalde-Herrá**, José Ángel Rodriguez-Getino*, \\ Juan Bautista Garcia-Casas*, Jorge Cipriano Diaz-Suarez*. \\ * Departamento de Medicina. Universidad de Oviedo, Oviedo. España. \\ ** Hospital Universitario Central de Asturias, Oviedo. España.
}

\section{Resumen}

La incidencia de las intoxicaciones agudas ha aumentado en los últimos años, y actualmente constituye aproximadamente el $2 \%$ de las atenciones sanitarias llevadas a cabo por los Servicios de Urgencias. El objetivo de este estudio es describir la frecuencia y características de las intoxicaciones atendidas en el Hospital Universitario Central de Asturias durante el año 2015 desde la perspectiva bioquímica-analítica, epidemiológica y médico-legal. Se realizó un estudio retrospectivo y un análisis descriptivo de las variables clínicas y sociodemográficas incluidas en el protocolo de intoxicación aguda a nivel nacional. Este hospital atendió 2478 casos de intoxicaciones agudas representando el 2,3\% de las urgencias atendidas y que corresponde a una incidencia de 764 casos/ 100000 habitantes/año con un rango de edad de menores de 1 año a mayores de 80 años. La edad media de los pacientes atendidos fue de 43,6 $(D E=16,6)$ años. El $59,4 \%$ de los pacientes eran varones con una edad media de $44(D E=16,8)$ años, las mujeres representaban el 43,1\% y su edad media era de $42,8(D E=16,5)$ años. El $47,2 \%$ de estas intoxicaciones ocurren durante el fin de semana y el $37,4 \%$ se dan entre junio y septiembre. La intencionalidad más frecuente es la intoxicación aguda voluntaria correspondiente al 83,2\% de los casos. Cabe destacar que el 16,8\% de los casos están referenciados en su historia clínica como intentos de suicidio. Los tóxicos más empleados son el etanol y las benzodiacepinas. Estas intoxicaciones son resueltas en el Servicio de Urgencias sin requerir ingreso hospitalario y poseen una tasa de mortalidad muy baja.

Palabras clave: Intoxicación aguda; Epidemiología; Toxicología; Intento suicidio; Intoxicación medicamentosa; Intoxicación alcohólica; Intoxicación por drogas abuso.

\begin{abstract}
The incidence of acute poisonings has increased in recent years and constitutes approximately $2 \%$ of the services provided by the Emergency Department currently.

The objective of this study is to describe the frequency and characteristics of the intoxications treated at the Central University Hospital of Asturias during 2015 from biochemical-analytical, epidemiological and medical-legal perspectives. We conducted a retrospective study and a descriptive analysis of the clinical and sociodemographic variables included in the acute intoxication (AI) protocol at the national level. This hospital treated 2,478 cases of acute poisoning, representing $2.3 \%$ of the emergencies treated and corresponding to an incidence of 764 cases/100,000 inhabitants/year with an age ranging from under 1 year to over 80 years. The average age of the patients was $43.6(S D=16.6)$ years. Of these patients, $59.4 \%$ were males with an average age of $44(S D=16.8)$ years, and women represented $43.1 \%$ with an average age of $42.8(S D=16.5)$ years. These intoxications have a frequency of $47.2 \%$ during the weekend, while $37.4 \%$ occur between June and September. Acute voluntary intoxication is the most frequent intentionality, corresponding to $83.2 \%$ of the cases. We must point out that the medical records register $16.8 \%$ of the cases as suicide attempts. Ethanol and benzodiazepines are the most commonly-used toxics. These intoxications are treated in the Emergency Department without requiring hospitalization and have a very low mortality rate. Keywords: Acute intoxication; Epidemiology; Toxicology; Suicide attempt; Alcohol intoxication; Drug overdose.
\end{abstract}




\section{Introducción}

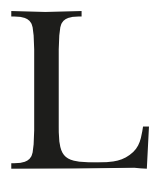

as intoxicaciones son tan antiguas como el ser humano, han estado presentes desde el inicio en la vida cotidiana de las personas condicionando sus hábitos de vida, su trabajo, sus momentos de ocio y diversión. Actualmente, la adquisición de estas nuevas sustancias tóxicas suele estar directamente relacionada con un mayor acceso a bienes de consumo (da Silva Moreira et al., 2010). La tecnología también favorece a la aparición de sustancias sintéticas ilegales cada vez más puras y nocivas que permiten crear y fomentar consumos patológicos a la sociedad. En los últimas décadas se ha demostrado un aumento en el número de intoxicaciones agudas (IA) en España constituyendo un problema de salud potencialmente grave (de Miguel-Bouzas et al., 2012).

Estas intoxicaciones agudas no sólo tienen implicación médica en su asistencia sanitaria, sino una serie de problemas económicos, socioculturales, demográficos, psicológicos y legales haciendo cada vez es más difícil su resolución, conllevando un problema social con repercusiones a gran escala (Caballero y Dorado, 1980; de la Fuente et al., 2006).

En un estudio sobre intoxicaciones agudas es importante conocer cuáles son los tóxicos implicados junto con su clínica característica y los antídotos o tratamientos específicos para cada una de ellas. (Caballero, Dorado y Alonso, 1981). Con estas premisas, se pueden llevar a cabo programas de prevención y formación de profesionales, así como dotar de soportes diagnósticos y terapéuticos a los servicios asistenciales hospitalarios y de Atención Primaria para la correcta realización de sus funciones asistenciales (Pastó Cardona, Martorell Puigserver, Mercadal Orfila, Machí Ribes y Jódar Massanès, 2007).

Como se ha indicado, las intoxicaciones agudas por sí mismas constituyen un fenómeno social en toda su extensión ya que de las conductas de los consumidores de sustancias tóxicas se derivan diversos problemas a la población general, por lo tanto se deben realizar estudios para conocer su epidemiología (Caballero, Gutiérrez y Dorado, 1987).

Los estudios epidemiológicos sobre intoxicaciones agudas publicados en los años 80 y 90 en todo el mundo, presentaban grandes diferencias en cuanto al diseño y a la metodología empleada, por lo tanto, la comparación de los resultados obtenidos y el análisis evolutivo era prácticamente imposible de establecer (Camí, Frati y Martín, 1980; Duce Tello et al., 1998).

En las últimas décadas, esta tendencia ha cambiado, se han realizado estudios multicéntricos, entre los que destacan dos estudios SEMESTOX e HISPATOX, realizados a nivel nacional entre 2003 y 2008 donde colaboraron los Servicios de Urgencias hospitalarias de diferentes Comunidades Autónomas y permiten tener una visión epidemioló- gica global de esta patología en todo el territorio nacional (Burillo-Putze et al., 2003; Burillo Putze et al., 2008).

Además de las características e incidencia de las intoxicaciones agudas, también interesa conocer su evolución en el tiempo, por lo que en determinadas áreas sanitarias se realizan estudios de series temporales de vigilancia epidemiológica en intoxicación aguda (VEIA) que se actualizan periódicamente manteniendo su validez (Caballero Vallés et al., 2008; Dorado et al., 1992; Dorado, Martín, Sabugal y Caballero, 1996).

La mayoría de los trabajos revisados son de los Servicios de Urgencias Hospitalarios, ya que actualmente es donde se realiza la atención sanitaria inicial y fundamentalmente de estos pacientes aunque cabe destacar que los primeros estudios sobre intoxicaciones agudas dentro del ámbito hospitalario se realizaron en los Servicios de Cuidados Intensivos para poner de manifiesto las medidas de tratamiento realizadas (Amigo-Tadín, Nogué-Xarau y Miró- Andreu, 2010; Duce Tello et al., 1998; Henderson, Wright y Pond, 1993; Medina, Fuentes, Suárez, Arranz y Ochoa, 2008).

Centrándose en el entorno más cercano, el Principado de Asturias, se han publicado algunos artículos aislados (Barraca de Ramos et al., 1991; González-Fernández y Alonso-Fernández, 2009; Rodríguez Getino e Hinojal Fonseca, 1994) que ayudaron a conocer la tendencia en esta comunidad en materia de intoxicaciones hace unos años, por lo que se quiere estudiar y comparar en la medida de lo posible, los resultados actuales.

Es útil explicar que en las intoxicaciones agudas, suele existir una relación casi siempre lineal entre la exposición a una sustancia, los valores analíticos detectados en las muestras biológicas y la aparición de síntomas y signos de enfermedad (Borrell, et al., 2001; Carpintero Escudero et al., 2000).

El objetivo de este estudio es conocer la frecuencia de las intoxicaciones agudas atendidas en el Servicio de Urgencias de un hospital de tercer nivel español para determinar las características analíticas, socioepidemiológicas, así como, las repercusiones médico-legales.

\section{Material y métodos}

Es un estudio descriptivo, retrospectivo llevado a cabo en el Área del Laboratorio de Medicina del Hospital Universitario Central de Asturias, hospital de tercer nivel y referencia en la Comunidad Autónoma, que tiene 1.039 camas y da cobertura al Área Sanitaria IV con 324.218 habitantes.

Los datos del estudio proceden de una revisión de los resultados analíticos realizados desde el 1 de enero al 31 de diciembre de 2015 en el Laboratorio de Medicina mediante el sistema informático del laboratorio (GestLab), para identificar a los pacientes cuyos valores analíticos de 
etanol o drogas de abuso superaban el límite de referencia por una intoxicación aguda. Este diagnóstico comprende la exposición al tóxico, junto con una clínica compatible en el sujeto y/o confirmación analítica mediante el estudio toxicológico (cuantificación de etanol en sangre y/o la detección de drogas en orina). Las analíticas son solicitadas por los profesionales del Servicio de Urgencias que bajo su criterio médico consideran necesarias estas pruebas complementarias.

Los límites de referencia son establecidos específicamente para cada sustancia concreta. La determinación de etanol se realiza mediante la técnica enzimática automatizada y es un resultado cuantitativo por lo que se considera IA a partir de $10 \mathrm{mg} / \mathrm{dL}$ aunque los primeros síntomas clínicos se visualizan con 40-50 mg/dL. Las drogas de abuso son sustancias que se analizan de manera cualitativa, es decir, sólo existe el resultado dicotómico de positivo o negativo mediante el método inmunocromatográfico de flujo lateral. Un resultado positivo indica que se ha superado el "cut-off" específico de la sustancia, es decir, que la concentración de la droga está por encima de ese valor (Tabla 1).

Tabla 1. Valores del cut-off de las drogas de abuso detectadas en el screening.

\begin{tabular}{lc}
\hline Sustancia de abuso & Valores cut-off \\
\hline Anfetamina (AMP) & $1000 \mathrm{ng} / \mathrm{mL}$ \\
Metanfetamina (MET) & $1000 \mathrm{ng} / \mathrm{mL}$ \\
Barbitúricos (BAR) & $300 \mathrm{ng} / \mathrm{mL}$ \\
Metadona (MTD) & $300 \mathrm{ng} / \mathrm{mL}$ \\
Cocaína (COC) & $300 \mathrm{ng} / \mathrm{mL}$ \\
Éxtasis (MDMA) & $500 \mathrm{ng} / \mathrm{mL}$ \\
Antidepresivos tricíclicos (TAC) & $1000 \mathrm{ng} / \mathrm{mL}$ \\
Benzodiacepinas (BZO) & $300 \mathrm{ng} / \mathrm{mL}$ \\
Cannabis (THC) & $50 \mathrm{ng} / \mathrm{mL}$ \\
Opiáceos (MOP) & $300 \mathrm{ng} / \mathrm{mL}$ \\
\hline
\end{tabular}

Nota. * "Cut-off" es el valor de concentración de la droga por encima de la cual el resultado es positivo y es posible detectarlo en orina con el método analítico correspondiente, en este caso es mediante inmunocromatografía de flujo lateral.

Se revisaron las historias clínicas electrónicas de los pacientes seleccionados de manera individualizada y se cumplimentó una ficha anonimizando el registro de cada uno con un numero clave "ad hoc" (que no tenía relación ni con su número de historia clínica ni con la identidad del sujeto) que recogía las variables motivo de estudio según características personales e intencionalidad de los pacientes, la temporalidad de las mismas, el tóxico utilizado, las medidas terapéuticas empleadas y las repercusiones médico-legales de los resultados obtenidos. Este estudio fue aprobado por el Comité de Ética del Principado de Astu- rias y el investigador respetó todas las normas de confidencialidad requeridas. La revisión fue realizada por la misma persona.

Las IA en Urgencias se clasificaron en dos grupos diferentes en función de su intencionalidad recogida en la historia clínica del paciente: IA voluntarias, en las que el sujeto realiza un consumo voluntario de alcohol o drogas buscando sus efectos psicoactivos y los intentos de suicidio, donde el consumo del tóxico se realiza con intención suicida.

Para el análisis estadístico se utilizó el paquete estadístico IBM SPSS Statistics v21. Se ha realizado una descripción de las variables estudiadas utilizando los estadísticos correspondientes en función del tipo de variable. Se empleó la media y la desviación estándar en el caso de variables cuantitativas y la proporción sobre el total en el caso de las categóricas.

\section{Resultados}

Se consideró criterio de caso, las muestras analíticas cuyo resultado fue superior al límite de referencia establecido. Se atendieron 4.586 casos de IA a lo largo del año, de los cuales 2.478 fueron atendidas en el Servicio de Urgencias representando el 2,3\% de todas las urgencias atendidas en el hospital durante el año 2015. Los 2.108 casos restantes, corresponden a IA atendidas y analizadas de manera rutinaria en otros servicios intra y extrahospitalarios. De estos 2.478 casos atendidos en Urgencias, el 5,3\% $(\mathrm{n}=131)$ corresponden a pacientes que requirieron asistencia en dos o más ocasiones durante el mismo periodo. La incidencia de las intoxicaciones atendidas en el Servicio de Urgencias en relación a la población total del área IV es de 764 casos/100.000 habitantes/año con un rango de edad comprendido entre menos de 1 año y más de 80 años. La edad media de los pacientes atendidos fue de 43,6 $(D E=16,6)$ años. El 59,4\% de los pacientes eran varones $(\mathrm{n}=1.411)$ con una edad media en este caso de $44(D E=16,8)$ años, las mujeres $(\mathrm{n}=1.067)$ representaban el $43,1 \%$ y su edad media era de 42,8 $(D E=16,5)$ años (Figura 1).

En relación a los diversos grupos de edad se puede observar que las edades comprendidas entre 41- 60 años representan el 43,2\% ( $n=1.070)$ de los atendidos en Urgencias. El rango de edad más frecuente en mujeres es entre 31-50 años donde se localiza el 47,4\% ( $\mathrm{n}=506)$, en cambio los hombres lo presentan entre 41- 60 años (42,3\%; $\mathrm{n}=$ 597) manteniendo la misma proporción que el total de IA atendidas en Urgencias.

Las intoxicaciones por sustancias de abuso atendidas en el Servicio de Urgencias tienen una frecuencia del 47,2\% $(\mathrm{n}=1.170)$ durante el fin de semana, período comprendido de viernes a domingo. Un 37,4\% ( $\mathrm{n}=929)$ ocurre en verano (junio-septiembre) (Figuras 2A y 2B). 


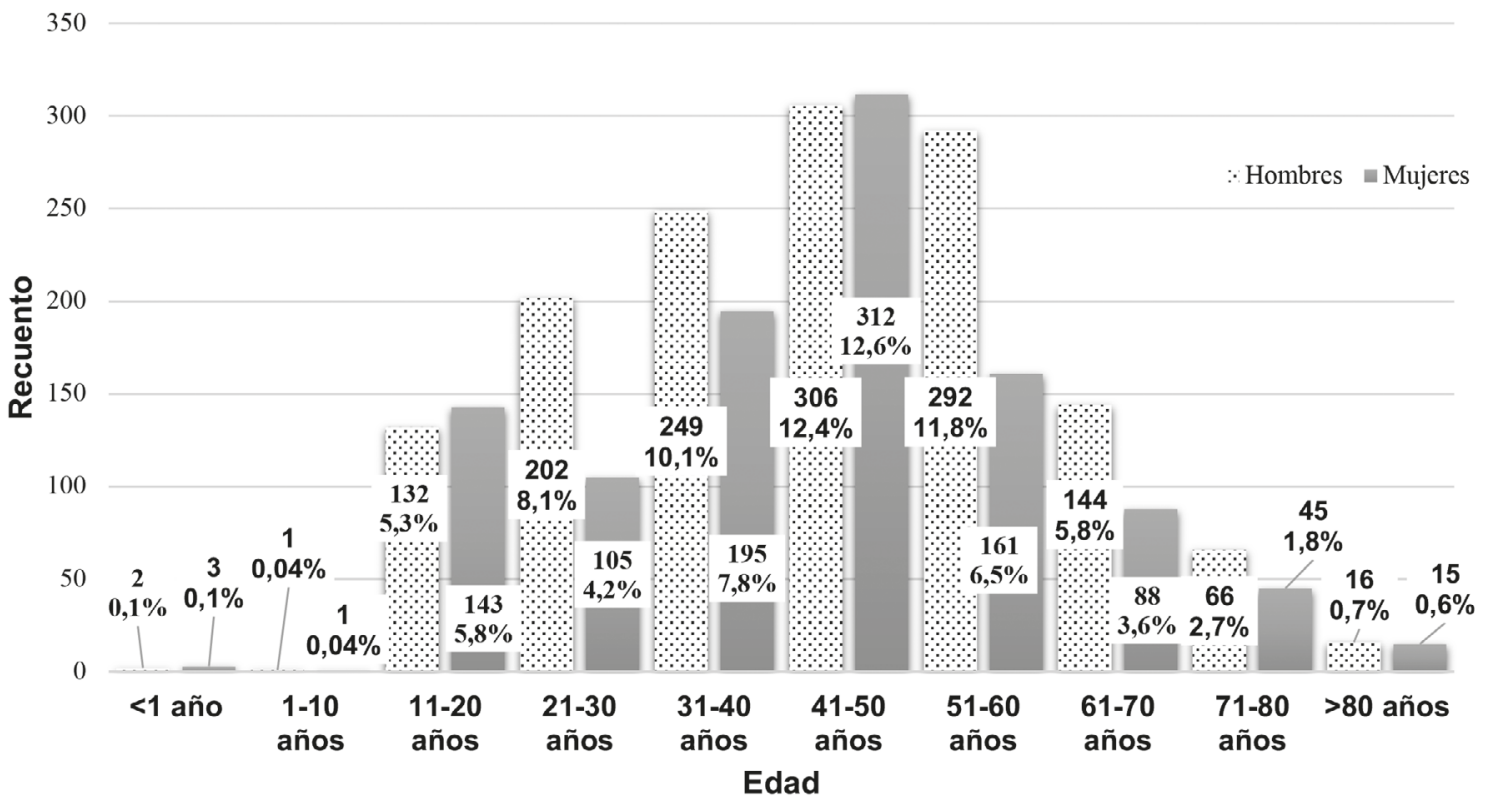

Figura 1. Distribución de las IA por edad y sexo.
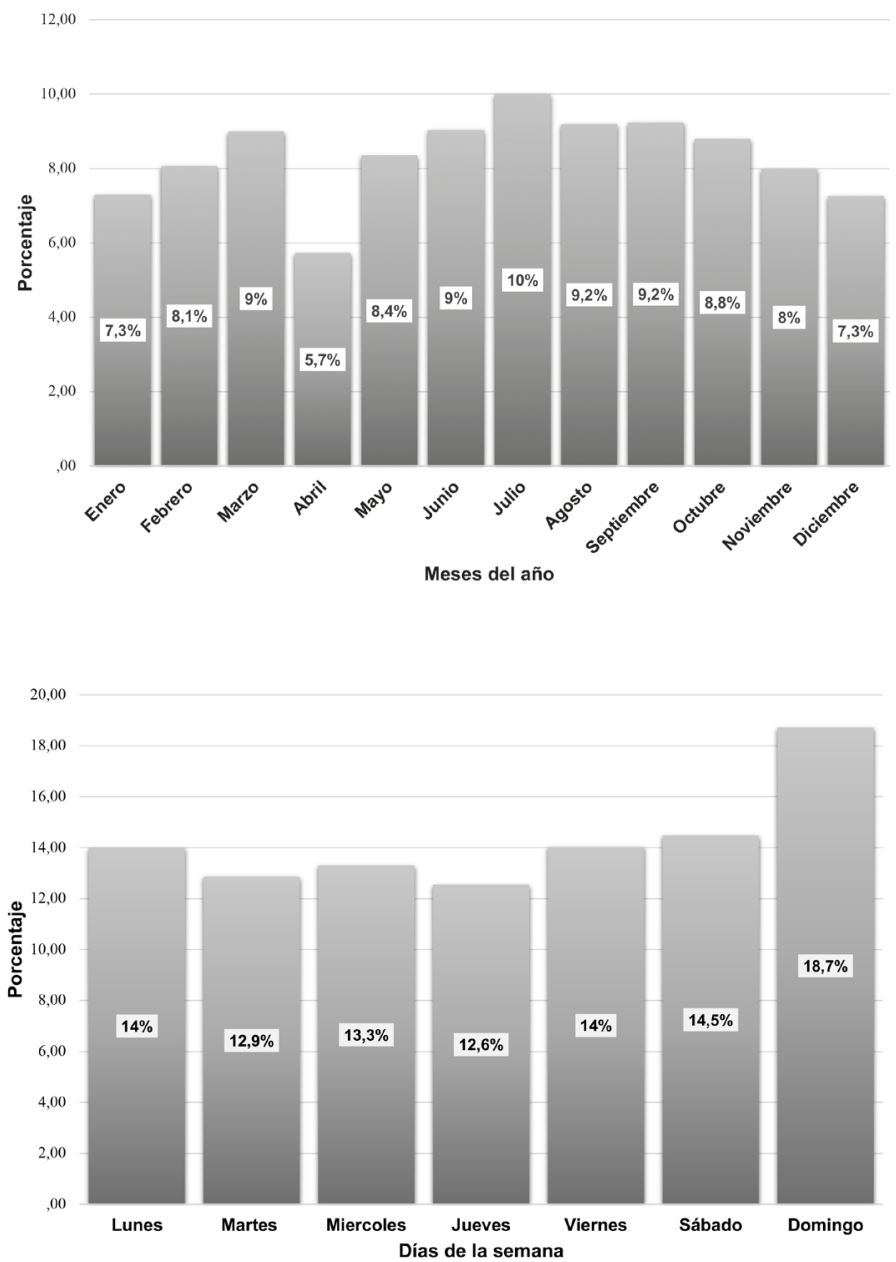

Figuras $2 A$ y $2 B$. Distribución de las IA según la fecha de admisión en Urgencias.
La intencionalidad más frecuente por parte del sujeto con respecto a las sustancias de abuso atendidas en el Servicio de Urgencias $(\mathrm{n}=2.478)$ es la IA voluntaria $(\mathrm{n}=$ 2.061 ) correspondiente al $83,2 \%$ de los casos. Cabe destacar la presencia de $16,8 \%(\mathrm{n}=417)$ referenciados como intentos de suicidio mediante la IA con fármacos, fundamentalmente benzodiacepinas u otros medios, como ahorcamiento o heridas incisas con armas blancas.

En el $52,8 \%(n=1.309)$ del total de casos atendidos en Urgencias existían antecedentes personales de patología psiquiátrica (establecidos según la clasificación DSM-V del año 2013, abarcando todos los comportamientos y signos clínicos visibles). Los predominantes fueron: trastornos adictivos y de consumo de sustancias ( $\mathrm{n}=297 ; 22,7 \%)$, trastornos de ansiedad $(\mathrm{n}=228 ; 17,4 \%)$ y trastornos depresivos $(\mathrm{n}=213 ; 16,2 \%)$.

Según los resultados analíticos toxicológicos positivos practicados a los pacientes con sospecha de IA atendidos en Urgencias se pueden diferenciar tres grupos. Un 34,6\% $(\mathrm{n}=856)$ corresponde a etanol en sangre, un 52,4\% ( $\mathrm{n}=$ 1.298) tenían screening cualitativo de drogas de abuso en orina y a un $13 \%(\mathrm{n}=324)$ presentaban IA por etanol y drogas de abuso en orina.

$\mathrm{Al}$ analizarse conjuntamente el primer y tercer grupo (n $=1.180$ ), el rango más frecuente de los valores cuantitativos de etanol es mayor de $100 \mathrm{mg} / \mathrm{dL}(\mathrm{n}=910)$ que corresponde a los pacientes que presentan signos y síntomas de depresión del sistema nervioso central. Se debe destacar que en las combinaciones de fármacos o drogas con etanol, el valor que se toma como referencia es la concentración de etanol, dándose por supuesto que el screening es positivo. 
Es reseñable que el 33,7\% $(\mathrm{n}=836)$ del total de pacientes intoxicados atendidos en Urgencias presentaban valores positivos en el screening cualitativo de benzodiacepinas en orina.

Si se analizan los tóxicos implicados en las IA según su intencionalidad, se observan que el 53,5\% ( $\mathrm{n}=223)$ de los pacientes que acuden por intentos de suicidio $(\mathrm{n}=417)$ presentan IA con benzodiacepinas, en cambio, el 39,6\% $(\mathrm{n}=816)$ de las intoxicaciones voluntarias $(\mathrm{n}=2.061)$ el tóxico detectado es el etanol (Tabla 2).

Las manifestaciones clínicas concomitantes reflejadas en la historia clínica de los intoxicados atendidos en Urgencias eran en el 34,3\% ( $n=208)$ manifestaciones tipo traumatológicas (heridas, fracturas, traumatismos craneoencefálicos, contusiones), que si bien no son consecuencia directa de las intoxicaciones, si que aparecen unidas a éstas por los accidentes y caídas que con frecuencia sufren estos pacientes. El 27,4\% ( $\mathrm{n}=166)$ de los casos presentaban clínica de tipo neurológico (mareos, inestabilidad en la marcha, disminución del nivel de conciencia). Con menor frecuencia, aparecen las manifestaciones tipo cardiológicas (palpitaciones, síncopes) o las manifestaciones digestivas (náuseas, vómitos).

El 38\% de las intoxicaciones agudas $(\mathrm{n}=942)$ fueron resueltas en el Servicio de Urgencias, bien recibiendo el alta a las pocas horas de su llegada o bien permaneciendo en boxes de observación. Un 19,7\% de los pacientes $(\mathrm{n}=489)$ precisó ingreso hospitalario y un $16,1 \%$ ( $\mathrm{n}=$ 399) de los casos necesitaron seguimiento por parte de su Centro de Salud Mental por este episodio agudo no por sus comorbilidades psiquiátricas ni trastornos adictivos. En nuestro estudio hubo un fallecimiento, implica una tasa de mortalidad del $0,1 \%$. Se trató de una poliintoxicación por drogas de abuso con intención suicida llevada a cabo por un varón politoxicómano (consumidor de varias clases de estupefacientes, sustancias o drogas) y con antecedentes de patología psiquiátrica, que fue traído desde Centro Penitenciario por la UVI móvil con bajo nivel de conciencia y shock séptico de etiología desconocida por lo que fue ingresado en UCI, donde falleció.

En un $4,8 \%$ de las intoxicaciones atendidas en Urgencias $(n=120)$ se emplearon medidas terapéuticas, requiriendo tratamiento farmacológico el 68,3\% de los pacientes $(n=82)$. Se utilizó flumazenilo en 41 casos de intoxicaciones agudas causadas por benzodiacepinas, naloxona en 8 intoxicaciones por opioides, combinación de flumazenilo y naloxona en 19 pacientes politoxicómanos y tiamina (vitamina B1) con piridoxina (vitamina B6) en 14 intoxicaciones etílicas. En el 31,7\% de los casos $(n=38)$

Tabla 2. Tóxicos individualizados y combinados detectados en los distintos tipos de IA atendidas en Urgencias.

\begin{tabular}{|c|c|c|c|}
\hline Sustancia de abuso & Intento de suicidio & Intoxicación voluntaria & Total \\
\hline Etanol & $40(9,6 \%)$ & $816(39,6 \%)$ & $856(34,5 \%)$ \\
\hline Anfetaminas (screening) & $1(0,2 \%)$ & o (o\%) & $1(0,1 \%)$ \\
\hline Antidepresivos tricíclicos (screening) & $3(0,7 \%)$ & $10(0,5 \%)$ & $13(0,5 \%)$ \\
\hline Barbitúricos (screening) & o (०\%) & $3(0,2 \%)$ & $3(0,1 \%)$ \\
\hline Benzodiacepinas (screening) & $223(53,4 \%)$ & $613(29,7 \%)$ & $836(33,7 \%)$ \\
\hline Cannabis (screening) & $7(1,7 \%)$ & $89(4,3 \%)$ & $96(3,8 \%)$ \\
\hline Cocaína (screening) & $4(1 \%)$ & $12(0,6 \%)$ & $16(0,6 \%)$ \\
\hline Metadona (screening) & o (०\%) & $5(0,2 \%)$ & $5(0,2 \%)$ \\
\hline Opiáceos (screening) & $1(0,2 \%)$ & $9(0,4 \%)$ & $10(0,4 \%)$ \\
\hline Metanfetaminas (screening) & o (०\%) & $1(0,1 \%)$ & $1(0,1 \%)$ \\
\hline Fármaco + droga ilegal & $35(8,4 \%)$ & $132(6,4 \%)$ & $167(6,7 \%)$ \\
\hline Fármaco + multidroga & $10(2,4 \%)$ & $51(2,5 \%)$ & $61(2,5 \%)$ \\
\hline Multifármacos & $15(3,6 \%)$ & $41(2 \%)$ & $56(2,3 \%)$ \\
\hline Multifámacos + droga ilegal & $1(0,2 \%)$ & $10(0,5 \%)$ & $11(0,4 \%)$ \\
\hline Multifármacos + multidrogas & $2(0,5 \%)$ & $2(0,1 \%)$ & $4(0,2 \%)$ \\
\hline Multidrogas & $1(0,2 \%)$ & $17(0,8 \%)$ & $18(0,7 \%)$ \\
\hline Alcohol + fármaco & $57(13,7 \%)$ & $116(5,6 \%)$ & $173(7 \%)$ \\
\hline Alcohol + multifármacos & $2(0,5 \%)$ & $1(1 \%)$ & $3(0,1 \%)$ \\
\hline Alcohol + droga ilegal & $5(1,2 \%)$ & $64(3,1 \%)$ & $69(2,8 \%)$ \\
\hline Alcohol + multidrogas & o (o\%) & $15(0,7 \%)$ & $15(0,6 \%)$ \\
\hline Alcohol + fármaco + droga ilegal & $8(1,9 \%)$ & $39(1,9 \%)$ & $47(1,9 \%)$ \\
\hline Alcohol + fármaco + multidrogas & $2(0,5 \%)$ & $15(0,7 \%)$ & $17(0,7 \%)$ \\
\hline Total & 417 & 2061 & 2478 \\
\hline
\end{tabular}




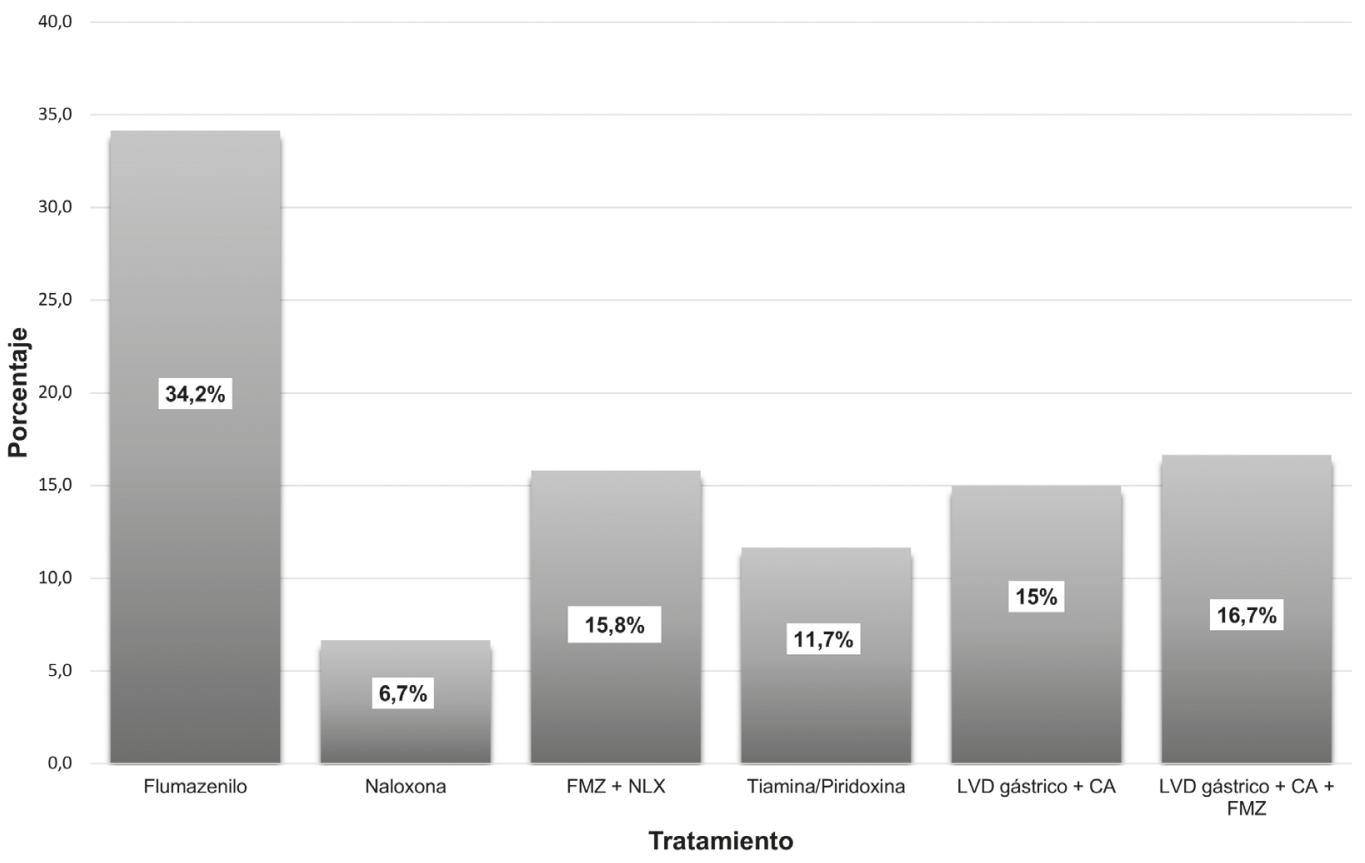

Figura 3. Tratamiento empleado en Urgencias para la resolución de las IA.

fue preciso realizar de manera conjunta un lavado gástrico con administración de carbón activado con/sin perfusión de flumazenilo (Figura 3).

Cabe destacar que sólo un $0,8 \%(\mathrm{n}=19)$ de estos resultados analíticos positivos para las diferentes intoxicaciones por abuso de sustancias pasan a ser muestras requeridas judicialmente y por tanto con implicaciones médico-legales.

\section{Discusión}

Los resultados obtenidos en este estudio muestran 2.478 intoxicaciones por sustancias de abuso atendidas en el Servicio de Urgencias que desde el punto de vista analítico presentan un predominio por etanol y benzodiacepinas. A nivel sociodemográfico las primeras corresponden con varones de edades comprendidas entre los 41-60 años y las segundas con mujeres en la tercera o cuarta década de la vida. Dentro de las repercusiones médico-legales de estas IA cabe destacar que sólo un $0,8 \%$ de los resultados analíticos positivos fueron requeridos judicialmente al estar implicados en diversos procedimientos.

Es preciso mencionar que el Hospital Universitario Central de Asturias, es el referente a nivel de la Comunidad Autónoma y da asistencia sanitaria a una población de 324.218 habitantes, distribuidos en 20 concejos incluyendo Oviedo a lo largo del área IV. Estos valores suelen aumentar porque, a él acuden pacientes de las diversas áreas sanitarias en un número aproximado de 3.000 personas por lo que alguna de las intoxicaciones que no requieran atención sanitaria urgente hospitalaria pueden ser atendidas en los Puntos de Atención Continuada.
La incidencia de intoxicaciones agudas que se presenta en el estudio, 764 casos/100.000 habitantes/año, es muy superior a la publicada previamente en estudios de similares características desarrollados en nuestro país. Este resultado parece estar en consonancia con lo que se observa de incremento de las IA a lo largo de las últimas décadas (Caballero, Dorado, Brusint, Jerez y Medina, 1999; Caballero Vallés et al., 2004; Dorado et al., 1992 y Dorado et al., 1996).

La edad media de los pacientes intoxicados, 44 años, es muy superior a la obtenida por otros autores (Fernández-Egido, García-Herrero, Romero-García y Marquina-Santos, 2008; García-Baró et al., 2005), lo que podría apuntar a que sujetos cada vez más mayores recurren al consumo de tóxicos de manera habitual.

Cabe destacar que el 2,9\% de las IA por etanol ocurren en sujetos adolescentes (14-15 años), valor muy inferior al $8,2 \%$ correspondiente a la tasa de consumo de alcohol en el nuestro medio en sujetos con las mismas edades (Bousoño et al., 2019) lo que apuntaría a lo que dicen algunos autores de que el consumo habitual de sustancias tóxicas está relacionado directamente con una mayor disponibilidad económica por parte del sujeto (Díaz Geada, Busto Miramontes y Caamaño Isorna, 2018).

La distribución de las IA en función del sexo confirmaría el predominio del sexo masculino en el total de intoxicaciones donde corresponde en nuestro estudio al 59,4\%, al igual que ocurre en la mayoría de los trabajos publicados tanto en España como en el extranjero (Clemente Rodríguez et al., 2010; da Silva Moreira et al., 2010; Fernández et al., 2003; Ferrer et al., 2005). 
Las IA voluntarias sufren un incremento muy importante durante el fin de semana (viernes, sábado y domingo) al corresponder con un $47,2 \%$ y un $37,4 \%$ en los meses de vacaciones fundamentalmente entre junio y septiembre por ser las épocas festivas, y posiblemente por aumentar las actividades lúdico-recreativas, tal y como apuntan muchos autores de que el consumo de las sustancias de abuso se asocia a momentos de ocio (Burillo-Putze et al., 2003; Echarte et al., 2005).

El porcentaje de intentos de suicidio $(16,8 \%)$ es similar al de otros estudios publicados y corresponde fundamentalmente a IA medicamentosas donde están presentes las benzodiacepinas (Lambert, Manel, Bellou. y el Kouch, 1997; Riquelme Rodríguez, Burillo-Putze, Jiménez Sosa y Hardisson De La Torre, 2001). Esta Comunidad Autónoma tiene una tasa de suicidio elevada con respecto al resto del país (Instituto Nacional de Estadística, 2016), pero los métodos empleados son ahorcamiento o precipitación (Iglesias García y Álvarez Riesgo, 1999). Los intentos de suicidio $(\mathrm{n}=417)$ son más frecuentes en mujeres ( $\mathrm{n}=252 ; 60 \%)$ y edades comprendidas entre $41-50$ años ( $\mathrm{n}$ $=135 ; 32,2 \%)$, tal y como se observa en estudios médicos publicados (Borrell et al., 2001; Fernández González, Sáiz Martínez, González G-Portilla, González Seijo y Bobes García, 2000; Jimenez-Trevino et al., 2012). Como medida preventiva a este fenómeno, en el Principado de Asturias se ha desarrollado el protocolo de detección y manejo de caso en personas con riesgo de suicidio. (Consejería de Sanidad y Servicio de Salud del Principado de Asturias, 2018).

En nuestro estudio, el tóxico implicado en el 47,6\% de los casos corresponde al etanol, ya sea analizado de manera aislada o en combinación con otros fármacos, en los últimos 20 años siempre se ha considerado el tóxico implicado en la mayoría de las IA (de las Cuevas, Sanz, de la Fuente, Cabrera y Mateos, 1999; García del Pozo et al., 2004). La segunda sustancia son las benzodiacepinas, fármacos que necesitan receta médica pero que son muy accesibles a la población y están presentes de forma aislada en el 33,7\% de las IA (Bugarin, Galego, Gude, García y Galban, 2000; Carpintero Escudero et al., 2000; Fernández, Sertral, Bermejo y Tabernero, 2005).

Algunos de estos pacientes $(n=606)$ al acudir al Servicio de Urgencias presentan una lesión concomitante, muchas veces han ocurrido de manera fortuíta y otras son intencionadas, destacan el TCE con/sin heridas y cefalea postraumáticas $(\mathrm{n}=208 ; 34,3 \%)$ y patología neurológica $(\mathrm{n}=166$; $27,4 \%$ ) que no difieren de las encontradas en estudios similares otras poblaciones españolas (Medina et al., 2008; Pascual Catalán, Fuentes Solsona, Castellano Arroyo, Ferrer Dufol y López Lancis, 1992; Pinillos, Grijalba y Alfaro, 2003).

La mejora en las técnicas de determinación analítica ha permitido que aumente el número de exámenes complementarios realizados a estos pacientes y se obtengan resultados con mayor fiabilidad que en épocas pasadas. Al
34,6\% de los casos se determinó el nivel de etanol en sangre, al 52,4\% se le realizó el screening cualitativo de drogas en orina y al $13 \%$ de los pacientes fueron sometidos a ambas determinaciones. Con estos datos, cabe destacar que el rango de resultados cuantitativos analizados por el método enzimático automatizado, más frecuentemente detectados en pacientes con IA por etanol es mayor de 100mg/ dL (36,7\%) cuyos signos y síntomas clínicos corresponden con depresión del sistema nervioso central manifestándose como letargia o disminución reflejos (Aragón, Miquel, Correa y Sanchis-Segura, 2002; Bajo Bajo et al., 1999).

En un $4,8 \%$ de las intoxicaciones atendidas $(n=120)$ se emplearon medidas terapéuticas. El 68,3\% de los pacientes $(\mathrm{n}=82)$ requirió tratamiento farmacológico. Se utilizó flumazenilo en 41 casos de intoxicaciones agudas causadas por benzodiacepinas, naloxona en ocho intoxicaciones por opioides, combinación de flumazenilo y naloxona en 19 pacientes politoxicómanos y tiamina (vitamina B1) con piridoxina (vitamina B6) en 14 intoxicaciones etílicas. El $31,7 \%$ de los casos $(n=38)$ fue preciso realizar de manera conjunta un lavado gástrico con administración de carbón activado con/sin perfusión de flumazenilo siguiendo las recomendaciones actuales (Benson et al., 2013; Burillo-Putze et al., 2003; Chyka et al., 2005).

Las acciones finales llevadas a cabo por parte del Servicio de Urgencias $(n=2.478)$ más frecuentes son observación domiciliaria/alta $(\mathrm{n}=942 ; 38 \%)$, ingreso $(\mathrm{n}=489$; $19,7 \%$ ) ya sea en planta o en la UCI dependiendo de la etiología de la IA y por último, seguimiento por parte de su Centro de Salud Mental ( $\mathrm{n}=399 ; 16,1 \%$ ) porque el $52,8 \%$ de los pacientes atendidos $(n=1309)$ tienen antecedentes de patología psiquiátrica previa. Únicamente hubo un caso de éxitus debido a poliintoxicación por drogas de abuso. El porcentaje de ingresos hospitalarios es menor que el observado en otros estudios (Hermida, Fernández, Ferrer, Bermejo y Tabernero, 2003).

Con respecto a la problemática jurídico-legal aplicable en estos casos de IA, hay que reseñar que 19 casos están implicados en delitos contra la Seguridad Vial tipificados en la Ley 6/2014 de 7 de abril, de los cuales el 84,2\% (n = 16) tenia la tasa de alcoholemia en sangre por encima de $0,5 \mathrm{~g} / \mathrm{L}$, lo que conllevará a multas de 500 euros y retirada de 4 puntos del carnet de conducir.

\section{Conclusiones}

La incidencia hallada en nuestro estudio es superior a la publicada previamente en concordancia con la tendencia de los últimos 20 años. El porcentaje de varones intoxicados que acuden al Servicio de Urgencias es aproximadamente del $60 \%$ similar a lo detectado en estudios anteriores.

La tendencia de estas intoxicaciones agudas ocurre durante los fines de semana y en los meses de periodo vaca- 
cional (junio-septiembre) ocurriendo lo mismo en nuestro caso. El número de intentos de suicidio que corresponden a un $16,8 \%$ de los pacientes que acuden para recibir atención hospitalaria urgente es similar a lo recogido anteriormente. El tóxico más utilizado ha sido el etanol siguiendo la tendencia de las últimas décadas, seguido de las benzodiacepinas a pesar de ser necesaria receta médica. Los resultados toxicológicos cuantitativos llevados a cabo como pruebas complementarias reflejaron que el $36,7 \%$ de las intoxicaciones por etanol poseían unos valores en sangre superiores a $100 \mathrm{mg} / \mathrm{dL}$ y que implican síntomas de depresión sistema nervioso central.

Los pacientes presentaban mayoritariamente manifestaciones traumatológicas a nivel de cabeza y cuello como traumatismos craneoencefálicos ocurridos de manera fortuita, seguidos de las neurológicas. El 54,1\% de los casos fueron solucionados en el área de Urgencias bien con observación/alta domiciliaria o seguimiento en el CSM sin precisar ingreso hospitalario. La mortalidad obtenida fue del $0,1 \%$ muy inferior a lo publicado últimamente.

\section{Organismos colaboradores}

El Servicio de Urgencias del Hospital Universitario Central de Asturias.

\section{Aspectos éticos}

Para la realización de todo el estudio, se tuvo presente, en todo momento, la legislación vigente en materia de investigación clínica establecida en la Declaración de Helsinki, en el Convenio del Consejo de Europa relativo a los derechos humanos y la biomedicina, en la Declaración Universal de la UNESCO sobre los derechos humanos. Cumpliéndose, de igual modo, con los requisitos establecidos en la legislación española en el ámbito de la investigación médica, la protección de datos de carácter personal y la bioética, con la Ley 14/2007, de julio de Investigación Biomédica y los demás requisitos establecidos por la legislación española al respecto. El estudio ha sido sometido para su aprobación al Comité de Ética e Investigación Clínica del Hospital Universitario Central de Asturias (HUCA).

\section{Conflicto de intereses}

Los autores no tienen conflictos de intereses que declarar.

\section{Bibliografía}

Amigo-Tadín, M., Nogué-Xarau, S. y Miró-Andreu, O. (2010). Presentación clínica, actitud terapéutica y evolución de las intoxicaciones agudas tratadas con carbón activado: ¿existen diferencias entre hombres y mujeres? Enfermería Clínica, 20, 273-279. doi:10.1016/j. enfcli.2010.06.003.

Aragón, C., Miquel, M., Correa, M. y Sanchis-Segura, C. (2002). Alcohol y metabolismo humano. Adicciones, 14 (Supl. 1), 23-42. doi:10.20882/adicciones.541.

Bajo Bajo, A., Santos Pérez, M. E., Sanz Ortega, F., Zapico Álvarez, N., Okatsu, K. T., García Pérez, A. y Borrás Beato, R. (1999). Estudio epidemiológico sobre intoxicaciones agudas y dotación de botiquines de antídotos. Anales de Medicina Interna, 16, 285-289.

Barraca de Ramos, R., Alonso Calo, L., Del Busto Prado, F., Ibarra Peláez, A., Menéndez Fernández, J. y Álvarez García, A. J. (1991). Aproximación epidemiológica a las intoxicaciones agudas en Asturias. Revista de Sanidad e Higiene Pública, 65, 53-60.

Benson, B. E., Hoppu, K., Troutman, W. G., Bedry, R., Erdman, A., Höjer, J., ... European Association of Poisons Centers and Clinical Toxicologists. (2013). Position paper update: gastric lavage for gastrointestinal decontamination. Clinical Toxicology, 51, 140-146. doi:10.3109/1 5563650.2013 .770154$.

Borrell, C., Pasarin, M. I., Cirera, E., Klutke, P., Pipitone, E. y Plasència, A. (2001). Trends in young adult mortality in three european cities: Barcelona, Bologne and $\mathrm{Mu}-$ nich, 1986-1995. Journal of Epidemiology and Community Health, 55, 577-582. doi:10.1136/jech.55.8.577.

Bousoño, M., Al-Halabí, S., Burón, P., Garrido, M., Díaz-Mesa, E. M., Galván, G., ... Bobes, J. (2019). Predictive factors of alcohol consumption in adolescents: data from 1-year follow-up prospective study. Adicciones, 31, 52-63. doi:10.20882/adicciones.998.

Bugarin, R., Galego, P., Gude, F., García, A. y Galban, C. (2000). Estudio de intoxicaciones etílicas agudas en un servicio de urgencias. Anales de Medicina Interna, 17, 588 591.

Burillo-Putze, G., Munne, P., Dueñas, A., Pinillos, M. A., Naveiro, J. M., Cobo, J., ... the Clinical Toxicology Working Group, Spanish Society of Emergency Medicine (SEMESTOX). (2003). National multicentre study of acute intoxication in emergency departments of Spain. European Journal of Emergency Medicine, 10, 101-104. doi: 10.1097/00063110-200306000-00006.

Burillo-Putze, G., Munné Mas, P., Dueñas Laita, A., Trujillo Martín, M. M., Jiménez Sosa, A., Adrián Martín, M. J., ... Investigadores del Estudio HISPATOX. (2008). Intoxicaciones agudas: perfil epidemiológico y clínico, y análisis de las técnicas de descontaminación digestiva utilizadas en los servicios de urgencias españoles en el año 2006 -Estudio HISPATOX-. Emergencias, 20, 15-26.

Caballero, P. J. y Dorado, M. S. (1980). Factores epidemiológicos de la intoxicación. Medicine, 59, 3691-3699.

Caballero, P. J., Dorado, M. S. y Alonso, F. (1981). Intoxicación aguda: Estudio de 673 casos. Medicina Clínica, 77, 139-145. 
Caballero, P. J., Dorado, M. S., Brusint, B., Jerez, B. y Medina, M. (1999). Vigilancia epidemiológica de la intoxicación aguda 1997 (Estudio de 1.140 casos del área sur de la Comunidad de Madrid). Revista Clínica Española, 199, 424-430.

Caballero, P. J., Gutiérrez, F. y Dorado, M. S. (1987). Epidemiología de la intoxicaron aguda: Estudio comparativo 1979-1985 en el área sur de la Comunidad Autónoma de Madrid. Revista Clínica Española, 181, 334-339.

Caballero Vallés, P. J., Dorado Pombo, S., Díaz Brasero, A., García Gil, M. E., Yubero Salgado, L., Torres Pacho, N., ... Cantero Bengoechea, J. (2008). Vigilancia epidemiológica de la intoxicación aguda en el área sur de la Comunidad de Madrid: estudio VEIA 2004. Anales de Medicina Interna, 25, 262-268. doi:10.4321/S021271992008000600003.

Camí, J., Frati, M. y Martín, M. L. (1980). Intoxicación aguda en Barcelona. Epidemiología y consideraciones sobre su terapéutica. Medicina Clínica, 75, 287-291.

Carpintero Escudero, J. M., Ochoa Gómez, F. J., Ruiz Aspiazu, J. I., Bragado Blas, L., Palacios Marín, G., Ramalle-Gómara, E., ... Grupo de Toxicología de SEMES-La Rioja (2000). Prevalencia de las intoxicaciones agudas en Urgencias de La Rioja. Emergencias, 12, 92-97.

Chyka, P. A., Seger, D., Krenzelok, E. P., Vale, J.A., American Academy of Clinical Toxicology \& European Association of Poisons Centres and Clinical Toxicologists. (2005). Position paper: Single-dose activated charcoal. Clinical Toxicology, 43, 61-87.

Clemente Rodríguez, C., Echarte Pazos, J. L., Aguirre Tejedo, A., Puente Palacios, I., Iglesias Lepine, M.L. y Supervía Caparrós, A. (2010). Diferencias entre hombres y mujeres en las características de las intoxicaciones. Emergencias, 22, 435-440.

Consejería de Sanidad y Servicio de Salud del Principado de Asturias. (2018). Protocolo de detección y manejo de caso en personas con riesgo de suicidio. Asturias: Unidad de Coordinación del Programa Marco de Salud Mental SESPA. Recuperado de https://www.astursalud.es/documents /31867/225127/PROTOCOLO+SUICIDIO+def.pdf/48775c44-b688-961e-be16-f81511d4eba2.

da Silva Moreira, C., Rezende Barbosa, N., Alves Vieira, R. C. P., Carvalho, M. R., Marangon, P. B., Carneiro Santos, P. L. y Teixeira Júnior, M. L. (2010). Análise retrospectiva das intoxicações admitidas no hospital universitario da UFJF no período 2000-2004. Ciencia \& Saúde Coletiva, 15, 879-888. doi:10.1590/S1413-8123201000030003.

de la Fuente, L., Brugal, M. T., Domingo-Salvany, A., Bravo, M. J., Neira-León, M. y Barrio, G. (2006). Más de treinta años de drogas ilegales en España: una amarga historia con algunos consejos para el futuro. Revista Española de Salud Pública, 80, 505-520.

de las Cuevas, C., Sanz, E. J., de la Fuente, J., Cabrera, C. y Mateos, A. (1999). Prescribed daily doses and risk factors associated with the use of benzodiazepines in primary care. Pharmacoepidemiology and Drug Safety, 8, 207-216. doi:10.1002/(SICI) 10991557(199905/06) 8:3<207::AID-PDS421>3.0.CO;2-1.

de Miguel-Bouzas, J. C., Castro-Tubío, E., Bermejo-Barrera, A. M., Fernández-Gómez, P., Estévez-Núñez, J. C. y Tabernero-Duque, M. J. (2012). Estudio epidemiológico de las intoxicaciones agudas atendidas en un hospital gallego entre 2005 y 2008. Adicciones, 24, 239-246. doi:10.20882/adicciones.95.

Díaz Geada, A., Busto Miramontes, A. y Caamaño Isorna, F. (2018). Alcohol, tobacco and cannabis consumption in adolescents from a multicultural population (Burela, Lugo). Adicciones, 30, 264-270. doi:10.20882/adicciones.915.

Dorado, M. S., Álvarez, R., Caballero, P. J., Medina, J., Casanova, C. y Granado, J. A. (1992). Epidemiología de la intoxicación aguda: Estudio de 815 casos habidos en 1990 en el área sur de la Comunidad de Madrid. Revista Clínica Española, 191, 131-136.

Dorado, M. S., Martín, J., Sabugal, G. y Caballero, P. J. (1996). Epidemiología de la intoxicación aguda: estudio de 613 casos habidos en 1994 en el área sur de la Comunidad de Madrid. Revista Clínica Española, 196, $150-156$.

Duce Tello, S., López Martínez, E., Navas Serrano, V., Piqueras Villaidea, M., Jara Peñacoba, M., Servián Carroquino, R., ... Abril García, A. (1998). Intoxicaciones medicamentosas voluntarias atendidas en un Servicio de Urgencias. Emergencias, 10, 225-233.

Echarte, J. L., Iglesias, M. L., Hernández, E., García, L., Orriols, M., Villar, J., ... Skaf, E. (2005). Registro de las intoxicaciones agudas en un Servicio de Urgencias hospitalario. Revista de Toxicología, 22, 116.

Fernández, P., Ortega, M., Bermejo, A. M., Tabernero, M. J., López-Rivadulla, M. y Concheiro, M. E. (2003). Intoxicaciones agudas en Santiago de Compostela en un período de cuatro años. Revista de Toxicología, 20, 216220.

Fernández, P., Sertral, R., Bermejo, A. M. y Tabernero, M. J. (2005). Intoxicaciones agudas por psicofármacos y drogas de abuso en Pontevedra durante el año 2001. Revista de Toxicología, 22, 37-40.

Fernández-Egido, C., García-Herrero, G., Romero-García, R. y Marquina-Santos, A. J. (2008). Intoxicaciones agudas en las urgencias extrahospitalarias. Emergencias, 20, 328-331.

Fernández González, C., Sáiz Martínez, P. A., González G-Portilla, M. P., González Seijo, J. C. y Bobes García, J. (2000). Tentativa suicida versus intención suicida: un estudio de las características diferenciales. Actas Españolas de Psiquiatría, 28, 224-230.

Ferrer, A., Royo, R., Rivas, M., Menao, S., Moreno, M. y Civeira, E. (2005). Perfil de las intoxicaciones agudas en el 
Servicio de Urgencias del Hospital Clínico de Zaragoza. Toxicología, 22, 121.

García-Baró, M., Lasarte-Turumbay, L., Prieto-Ferrer, M., Montiel, J. A., Lloret, J. y Marruecos, L. (2005). Intoxicaciones agudas del adulto. Epidemiología en un Servicio de Urgencias. Toxicología, 22, 119-120.

García del Pozo, J., de Abajo Iglesias, F. J., Carvajal García-Pando, A., Montero Corominas, D., Madurga Sanz, M. y García del Pozo, V. (2004). Utilización de ansiolíticos e hipnóticos en España (1995-2002). Revista Española de Salud Pública, 78, 379-387.

González-Fernández, D. y Alonso-Fernández, M. (2009). Intoxicaciones agudas en un Servicio de Urgencias. Estudio descriptivo en el Área Sanitaria III de Asturias. Revista de Toxicología, 26, 122-127.

Henderson, A., Wright, M. y Pond, S. M. (1993). Experience with 732 acute overdose patients admitted to an intensive care unit over six years. Medical Journal of Australia, 158, 28-30.

Hermida, I., Fernández, P., Ferrer, A., Bermejo, A. M. y Tabernero, M. J. (2003). Perfil psicosocial de pacientes ingresados por intoxicación aguda voluntaria. Revista de Toxicología, 20, 33-37.

Iglesias García, C. y Álvarez Riesgo, J. A. (1999). Un estudio del suicidio en Asturias: incremento de la frecuencia en las dos últimas décadas. Actas Españolas de Psiquiatría, 27, 217-222.

Instituto Nacional de Estadística. (2016). Estadística del suicidio en España 2015. Recuperado de http://www. ine.es $/$ jaxi $/$ tabla.do? type $=$ pcaxis \&path $=/ \mathrm{t} 15 / \mathrm{p} 417 /$ a2014/10/\&file=06004.px.

Jimenez-Trevino, L., Saiz, P. A., Corcoran, P., Garcia-Portilla, M. P., Buron, P., Garrido, M., ... Bobes, J. (2012). The incidence of hospital-treated attempted suicide in Oviedo, Spain. Crisis, 33, 46-53. doi:10.1027/0227$5910 / \mathrm{a} 000094$.
Lambert, H., Manel, J., Bellou, A. y el Kouch, S. (1997). Morbidity and mortality from acute drug poisoning in France. La Revue du Practicien, 47, 716-720.

Ley $6 / 2014$, de 7 de abril, por la que se modifica el texto articulado de la Ley sobre Tráfico, Circulación de Vehículos a Motor y Seguridad Vial, aprobado por el Real Decreto Legislativo 339/1990, de 2 de marzo. Boletín Oficial del Estado (BOE), 85, de 8 de abril de 2014, 2950829528.

Medina, L., Fuentes, M. E., Suárez, J. P., Arranz, M. I. y Ochoa, E. (2008). Epidemiología de las intoxicaciones medicamentosas durante un año en el Hospital Universitario Ramón y Cajal. Revista Clínica Española, 208, 432436. doi:10.1157/13127603.

Pascual Catalán, A., Fuentes Solsona, F., Castellano Arroyo, M., Ferrer Dufol, A. y López Lancis, A. (1992). Estudio epidemiológico de las intoxicaciones agudas en la población de Zaragoza. Anales de Medicina Interna, 9, 381-385.

Pastó Cardona, L., Martorell Puigserver, C., Mercadal Orfila, G., Machí Ribes, J. J. y Jódar Massanès, R. (2007). Intoxicaciones agudas en el servicio de urgencias de un hospital universitario de nivel III: cambios producidos en los últimos 10 años. Revista de Toxicología, 24, 36-41.

Pinillos, M. A., Grijalba, A. y Alfaro, J. (2003). Situación de las intoxicaciones en Navarra. Anales del Sistema Sanitario de Navarra, 26 (Supl. 1), 7-19.

Riquelme Rodríguez, A., Burillo-Putze, G., Jiménez Sosa, A. y Hardisson De La Torre, A. (2001). Epidemiología global de la intoxicación aguda en un área de salud. Atención Primaria, 28, 506-509.

Rodríguez Getino, J. A. e Hinojal Fonseca, R. (1994). Intoxicaciones agudas: estudio epidemiológico retrospectivo en un Área Asturiana (1985-1989). Revista de Toxicología, 11, 99-104. 